\title{
Structural insights into the mechanism of Plasmepsins from P. falciparum
}

$\underline{\text { Ishan Rathore }}^{1}$, Vandana Mishra ${ }^{1}$, Chandan Patel ${ }^{2}$, Huogen Xiao ${ }^{3}$, Rickey Yada ${ }^{4}$, Alla Gustchina ${ }^{5}$, Alexander Wlodawer ${ }^{5}$, Koushi Hidaka ${ }^{6}$, Prasenjit Bhaumik ${ }^{1}$

${ }^{1}$ Department Of Biosciences And Bioengineering, IIT-Bombay, Mumbai, India, 2Department of Chemistry, IIT-Bombay, Mumbai, India, ${ }^{3}$ Department of Molecular and Cellular Biology, University of Guelph, Ontario, Canada, ${ }^{4}$ Faculty of Land and Food Systems, University of British Columbia, Vancouver, Canada, ${ }^{5}$ Protein Structure Section, Macromolecular Crystallography Laboratory, National Cancer Institute, Frederick, United States, ${ }^{6}$ Division of Pharmaceutical Chemistry, Faculty of Pharmaceutical Sciences, Kobe Gakuin University, Kobe, Japan E-mail: ishanrathore21@gmail.com

Aspartic proteases are a family of enzymes with two catalytic aspartates in the active site and a conserved water molecule in between them, which is responsible for proteolytic action of enzyme. One of these aspartates, D215 is supposed to act as a catalytic acid and D32 as a base [1,2]. The reaction begins when D215 abstracts a proton from water, leading to nucleophilic attack on the peptide bond forming a tetrahedral intermediate. This tetrahedral intermediate is destabilized by the proton donation by D32, leading to peptide bond cleavage [3]. Nonetheless, the presence of differential charge on catalytic aspartates is not yet understood. Plasmepsins, which are pepsin-like aspartic proteases present in Plasmodium falciparum, engage in haemoglobin degradation and have emerged as attractive drug targets against malaria over the years. In our study, we are using Plasmepsin II (PMII) as a model system to decipher the mechanism of aspartic proteases, both functionally and structurally. Strategically, we have followed two different approaches, which includes the study of binding mode of different peptidomimetic inhibitors in the active site of PMII by co-crystallization of protein-inhibitor complexes as well as extensive mutagenesis studies.

We have solved three crystal structures of PMII bound to peptidomimetic KNI inhibitors (10006, 10772 and 10773), which allowed us to elucidate the mode of peptide binding in the active site. Interestingly, we have found that the minor modifications in the functional groups of these inhibitors alter their binding mode. Moreover, in the crystal structure of KNI10773 bound PMII, we have captured the catalytic water molecule, thus mimicking the peptide binding mode. In our all inhibitor bound structures, the active site aspartates are found to be non-planar which is unusual and has not been reported earlier. In another approach, based on our observations that the single aspartate mutants of D32A and D215A were still active, we have solved the crystal structure of D215A mutant. Another residue T218 that was proposed for charge protection on D215 was found to be non-essential and the T218A mutant was found to be active comparable to WT PMII, therefore, we have structurally characterized the T218A mutant. Both the mutant structures have helped in understanding the geometry of the residues in the active site. The detailed knowledge of the importance of residues in the active site will help in designing potent mechanism based inhibitors.

Plasmepsins are synthesized as inactive zymogens, and is activated on exposure to acidic condition. Small angle X-ray scattering experiments on Plasmepsin I (PMI) zymogen, indicate that the pro-plasmepsins form a stable S-shaped dimer and might be involved in the stability of the zymogen. We have solved the crystal structure of one of the plasmepsins HistoAspartic Protease (HAP) zymogen and have observed dynamic nature of the prosegment. In our analysis of crystal structures of other plasmepsin zymogens and MD simulation studies, the disruption of a single $\mathrm{H}$-bond results in breaking a chain of $\mathrm{H}$-bonding interactions. This leads to flexibility of the prosegment and rendering it more prone to hydrolysis.

[1] Davies, D. R. (1990). Annual review of biophysics and biophysical chemistry, 19, 189-215.

[2] Dunn, B. M. (20002). Chemical Reviews, 102, 4431-4458.

[3] Andreeva, N. S. \& Rumsh, L. D. (20001). Protein Science, 10, 2439-2450.

Keywords: Aspartic Proteases, Mechanism, Plasmepsin 